\title{
New representation of PV buses in the current injection Newton power flow
}

\author{
C.C. Oliveira ${ }^{a}$, A. Bonini Neto ${ }^{\text {b, } *}$, C.R. Minussi ${ }^{a}$, D.A. Alves ${ }^{a}$, C.A. Castro ${ }^{c}$ \\ ${ }^{a}$ São Paulo State University (UNESP), School of Engineering, Electrical Engineering Department, Ilha Solteira, SP, Brazil \\ b São Paulo State University (UNESP), School of Sciences and Engineering, Tupã, SP, Brazil \\ ${ }^{\mathrm{c}}$ University of Campinas (UNICAMP), Power System Department, SP, Brazil
}

\section{A R T I C L E I N F O}

Article history:

Received 22 August 2016

Received in revised form 26 October 2016

Accepted 2 January 2017

Available online 24 February 2017

\section{Keywords:}

Power flow

Current injection power flow

PV representation

Polar coordinates

\begin{abstract}
A B S T R A C T
This paper presents a comparative analysis of the influence of PV bus representation on the convergence characteristics of the Newton-Raphson Current Injection method. The methods used for solving the power flow problem are based on current injection equations written in polar coordinates. Several tests are presented to clarify the reasons of non-convergence and to provide a broad and fair comparison and evaluation of the performance of the PV bus representation schemes. Performance analyzes were conducted for 57-, 118- and 300-bus IEEE test systems and for two realistic Brazilian systems of 638 and 787 buses, corresponding to parts of the South-Southeast Brazilian system. Several R/X transmission line ratios and loading conditions were considered. The simulation results show that the proposed PV bus representation improves the convergence characteristics of the method.
\end{abstract}

(c) 2017 Elsevier Ltd. All rights reserved.

\section{Introduction}

The power flow tool is extensively applied in power systems planning and operation analyzes. It is also used to provide the initial conditions for several studies such as short-circuit, angle and voltage stability [1-3], among others. In real time operation, it is used for simulating transmission line contingencies and loading margin determination, requiring high computational processing time. Thus, there is a continuing search for methods that exhibit faster convergence and are also more robust and reliable.

Among the various numerical methods used for solving the power flow problem, Newton-Raphson iterative method is the most widely used, since it is more reliable and the number of iterations required for convergence is independent of the power system size. Both current and power injection power flow formulations can be written with voltages and admittances in either polar or rectangular coordinates. In some cases, hybrid formulations can also be used [3].

Considering the incorporation of FACTS devices and control strategies, current injection methods have been proposed in rectangular [4] and in polar [5] forms. Hybrids of current and power injection methods have also been proposed [6-9]. The use

\footnotetext{
* Corresponding author.

E-mail addresses: cout_cris@yahoo.com.br (C.C. Oliveira), bonini@tupa.unesp.br (A. Bonini Neto), minussi@dee.feis.unesp.br (C.R. Minussi), dalves@dee.feis.unesp.br (D.A. Alves), ccastro@ieee.org (C.A. Castro).
}

of rectangular coordinates results in a reduction of the effort to compute the Jacobian terms, since most of them remain constant during the iterative process. On the other hand, the use of polar coordinates leads to a smaller number of equations [10]. An augmented rectangular model in which bus voltages and current injections are retained in the state vector is presented in [11]. This approach shows to be advantageous when many zero-injection buses are present. In most of the current injection formulations, the appropriate representation of PV buses has been the main concern. Differently from PQ buses, for which the real and imaginary current mismatches are known, the reactive power mismatch $\Delta Q$ of a PV bus is unknown. Therefore, an additional equation for each $\mathrm{PV}$ bus is introduced in the rectangular formulation. The use of current injection mismatches with reactive mismatch $\Delta Q$ as a dependent variable, together with a voltage magnitude constraint equation, both written in rectangular coordinates are proposed in $[4,12,13]$. In [6] a hybrid formulation of current injection method written in polar coordinates, using a dependent variable $\Delta Q$ for each PV bus associated to an active power mismatch equation is proposed. A hybrid power flow method with PV buses represented by equations of active power mismatches using angle deviation as variable and PQ buses represented by equations of current injection written in rectangular coordinates are presented in $[7,14,15]$. A simplified Newton Raphson power flow solution method that uses nonlinear current mismatch equations written in polar coordinates is presented in [5]. A comparison is done with the power 
flow method using power mismatches also written in polar coordinates. The generated reactive powers of PV buses are recalculated alternately in the iterative process, i.e., during the iteration process the generated reactive power is kept unchanged, being recalculated before the next one.

The objective of this paper is to present a new formulation for representing PV buses to improve the convergence characteristics of the current injection models used in power flow analysis of electric power systems. The new formulation does not result in Jacobian matrix structure and size changes, leading only to little numerical differences in its elements. The performance of the proposed method is assessed by numerical simulations conducted for the 57-, 118- and 300-bus IEEE test systems and for two realistic Brazilian systems of 638 and 787 buses, corresponding to parts of the South-Southeast Brazilian system. The performance assessment is carried out considering different $\mathrm{R} / \mathrm{X}$ transmission line ratios and loading conditions. The voltage magnitudes of the critical bus obtained by each method are plotted as P-V curves to confirm that the converged state obtained by all methods is the same. The P-V curves are also used to clarify why the methods do not converge for specific $\mathrm{R} / \mathrm{X}$ transmission line ratios and loading conditions. The results obtained with the new representation of PV buses show that the convergence characteristics can be improved.

\section{Power flow in polar coordinates}

The objective of this section is to describe the power and current injection models used for the power flow analysis of electric power systems. In order to highlight their convergence characteristics and provide a fair comparison of the two models through simulations, full polar coordinates are used for both, i.e., current injections, bus voltages, injected powers and nodal admittance matrix elements are represented in polar coordinates.

\subsection{Newton Raphson power flow - formulation based on power injection}

In the standard power flow (SPF) formulation based on power injection, the active $\left(\Delta P_{k}(\boldsymbol{\theta},|\mathbf{V}|)\right)$ and reactive $\left(\Delta Q_{k}(\boldsymbol{\theta},|\mathbf{V}|)\right)$ power mismatch equations at bus $k$ take the following form [3],[16]:

$\Delta P_{k}(\boldsymbol{\theta},|\mathbf{V}|)=P_{k}^{s p}-P_{k}(\boldsymbol{\theta},|\mathbf{V}|)=0$
$\Delta Q_{k}(\boldsymbol{\theta},|\mathbf{V}|)=Q_{k}^{s p}-Q_{k}(\boldsymbol{\theta},|\mathbf{V}|)=0$

where $|\mathbf{V}|$ and $\boldsymbol{\theta}$ are the vectors of the nodal voltage magnitudes and phase angles respectively. The specified active and reactive powers are given by:

$P_{k}^{s p}=P_{\text {gen }, k}^{s p}-P_{\text {load }, k}^{s p}=\left|S_{k}^{s p}\right| \cos \left(\varphi_{k}\right)$

$Q_{k}^{s p}=Q_{\text {gen }, k}-Q_{\text {load }, k}^{s p}=\left|S_{k}^{s p}\right| \sin \left(\varphi_{k}\right)$

where $\left|S_{k}^{s p}\right|$ and $\varphi_{k}$ are the magnitude and angle of the net specified complex power injected at bus $k$ respectively, $P_{\text {gen }, k}^{s p}$ and $P_{\text {load }, k}^{s p}$ are, respectively, the specified real power generated and consumed for the load (PQ) and generation (PV) buses, and $Q_{\text {load.k }}^{s p}$ is the consumed reactive power specified for the $\mathrm{PQ}$ buses. In general, the generated reactive power $Q_{\text {gen, }}$ for a PQ bus is equal to zero or it has a fixed value. However, it is not known a priori for a PV bus, and its value is obtained after the convergence of (1). It may take any value within its maximum $\left(Q_{\text {gen }, k}^{\max }\right)$ and minimum $\left(Q_{\text {gen }, k}^{\min }\right)$ limits. The respective balance equation is not included in (1). On the other hand, if one of its limits is reached, the generated reactive power is fixed at the corresponding value and its balance equation is now included in (1) with $Q_{k}^{s p}$ given as in (2). The transmitted active $P_{k}(\boldsymbol{\theta},|\mathbf{V}|)$ and reactive $Q_{k}(\boldsymbol{\theta},|\mathbf{V}|)$ powers, considering bus voltages $\left(V_{k}\right.$ and $V_{i}$ ) and line admittances $\left(Y_{k i}\right)$ written in polar form, are calculated by [3]:

$$
\begin{aligned}
& P_{k}(\boldsymbol{\theta},|\mathbf{V}|)=\left|V_{k}\right| \sum_{i \in \kappa}\left|Y_{k i}\right|\left|V_{i}\right| \cos \left(\phi_{k i}-\theta_{k i}\right) \\
& Q_{k}(\boldsymbol{\theta},|\mathbf{V}|)=-\left|V_{k}\right| \sum_{i \in \kappa}\left|Y_{k i}\right|\left|V_{i}\right| \sin \left(\phi_{k i}-\theta_{k i}\right)
\end{aligned}
$$

where $\kappa$ is the set of buses directly connected to bus $k$ plus bus $k$ itself, $\left|V_{k}\right|,\left|V_{i}\right|, \theta_{k}$, and $\theta_{i}$ are the voltage magnitudes and angles at buses $k$ and $i,\left|Y_{k i}\right|$ and $\varphi_{k i}$ are the magnitude and angle of element $(k, i)$ of the nodal admittance matrix $\mathbf{Y}$, and $\theta_{k i}=\theta_{k}-\theta_{i}$ is the voltage phase angle difference between the buses $k$ and $i$. In a $n$-bus system with one slack bus, $n p q$ load buses (PQ buses) and $n p v$ generator buses (PV buses), there will be $(2 n p q+n p v)$ power mismatch equations, being $(n p v+n p q)$ corresponding to active power mismatches and $n p q$ corresponding to reactive power mismatches. In this case, the Taylor's series expansion of (1), neglecting the higher order terms, yields:

$$
\left[\frac{\Delta \mathbf{P}(\boldsymbol{\theta},|\mathbf{v}|)}{\Delta \mathbf{Q}(\theta,|\mathbf{V}|)}\right]=-\mathbf{J}\left[\frac{\Delta \theta}{\Delta|\mathbf{V}|}\right]=\left[\begin{array}{l|l}
\mathbf{H} & \mathbf{N} \\
\hline \mathbf{M} & \mathbf{L}
\end{array}\right]\left[\frac{\Delta \theta}{\Delta|\mathbf{V}|}\right]
$$

where $\boldsymbol{\Delta}|\mathbf{V}|$ and $\boldsymbol{\Delta} \boldsymbol{\theta}$ are, respectively, the vectors of the nodal voltage magnitude and phase angle corrections. The order of the Jacobian matrix is $(2 n p q+n p v)$ by $(2 n p q+n p v)$. The elements of the sub-matrices that compose the Jacobian $(\mathbf{J}), \quad \mathbf{H}=\partial \mathbf{P}(\boldsymbol{\theta},|\mathbf{V}|) / \partial \boldsymbol{\theta}$, $\mathbf{N}=\partial \mathbf{P}(\boldsymbol{\theta},|\mathbf{V}|) / \partial|\mathbf{V}|, \mathbf{M}=\partial \mathbf{Q}(\boldsymbol{\theta},|\mathbf{V}|) / \partial \boldsymbol{\theta}$ and $\mathbf{L}=\partial \mathbf{Q}(\boldsymbol{\theta},|\mathbf{V}|) / \partial|\mathbf{V}|$ are given by:

Submatrix H:

$H(k, i)=-\left|V_{k}\right|\left|V_{i}\right|\left|Y_{k i}\right| \sin \left(\phi_{k i}-\theta_{k i}\right) \quad$ for $k \neq i$

$H(k, k)=-Q_{k}-\left|V_{k}\right|^{2}\left|Y_{k k}\right| \sin \left(\phi_{k k}\right)$

Submatrix N:

$N(k, i)=\left|V_{k}\right|\left|Y_{k i}\right| \cos \left(\phi_{k i}-\theta_{k i}\right) \quad$ for $k \neq i$

$N(k, k)=\left(P_{k}+\left|V_{k}\right|^{2}\left|Y_{k k}\right| \sin \left(\phi_{k k}\right)\right) /\left|V_{k}\right|$

Submatrix M:

$M(k, i)=-\left|V_{k}\right|\left|V_{i}\right|\left|Y_{k i}\right| \cos \left(\phi_{k i}-\theta_{k i}\right) \quad$ for $k \neq i$

$M(k, k)=P_{k}-\left|V_{k}\right|^{2}\left|Y_{k k}\right| \cos \left(\phi_{k k}\right)$

Submatrix $\mathbf{L}$ :

$L(k, i)=-\left|V_{k}\right|\left|V_{i}\right|\left|Y_{k i}\right| \sin \left(\phi_{k i}-\theta_{k i}\right) \quad$ for $k \neq i$

$L(k, k)=\left(Q_{k}-\left|V_{k}\right|^{2}\left|Y_{k k}\right| \sin \left(\phi_{k k}\right)\right) /\left|V_{k}\right|$

For chosen initial values of $\boldsymbol{\theta}$ and $|\mathbf{V}|$, the mismatches are computed by (1). If their maximum absolute value is smaller than an adopted tolerance the solution was obtained. Otherwise, solve (4) to obtain the correction vectors $(\Delta \boldsymbol{\theta}$ and $\Delta|\mathbf{V}|)$ and update their values as follows:

$$
\begin{aligned}
& \boldsymbol{\theta}^{v+1}=\boldsymbol{\theta}^{v}+\boldsymbol{\Delta} \boldsymbol{\theta}^{v} \\
& |\mathbf{V}|^{v+1}=|\mathbf{V}|^{v}+\boldsymbol{\Delta}|\mathbf{V}|^{v}
\end{aligned}
$$

where $v$ is an iteration counter. 
2.2. Newton Raphson power flow - formulation based on current injection

In the power flow formulation based on current injection with balance equations written in polar coordinates, hereafter referred to as current injection power flow (CIPF), the complex current mismatch at a given bus $k$ is given by $[5,15]$ :

$\Delta F_{k}(\boldsymbol{\theta},|\mathbf{V}|)=\left(\frac{S_{k}^{s p}}{V_{k}}\right)^{*}-\sum_{i \in \kappa} Y_{k i} V_{i}=0$

whose real $\left(\Delta G_{k}(\boldsymbol{\theta},|\mathbf{V}|)\right)$ and imaginary $\left(\Delta H I_{k}(\boldsymbol{\theta},|\mathbf{V}|)\right)$ components are given by:

$\Delta G_{k}(\boldsymbol{\theta},|\mathbf{V}|)=\frac{\left|S_{k}^{s p}\right|}{\left|V_{k}\right|} \cos \left(-\varphi_{k}+\theta_{k}\right)-\sum_{i \in \mathcal{K}}\left|Y_{k i}\right|\left|V_{i}\right| \cos \left(\phi_{k i}+\theta_{i}\right)=0$
$\Delta H I_{k}(\boldsymbol{\theta},|\mathbf{V}|)=\frac{\left|S_{k}^{s p}\right|}{\left|V_{k}\right|} \sin \left(-\varphi_{k}+\theta_{k}\right)-\sum_{i \in \mathcal{K}}\left|Y_{k i}\right|\left|V_{i}\right| \sin \left(\phi_{k i}+\theta_{i}\right)=0$

The Newton Raphson linearized equation can be written as:

$\left[\begin{array}{c}\Delta \mathbf{G} \\ \Delta \mathbf{H I}\end{array}\right]=-\mathbf{J}_{\mathbf{I}}\left[\begin{array}{c}\Delta \theta \\ \Delta|\mathbf{V}|\end{array}\right]=\left[\begin{array}{ll}\mathbf{J}_{1} & \mathbf{J}_{2} \\ \mathbf{J}_{3} & \mathbf{J}_{4}\end{array}\right]\left[\begin{array}{c}\Delta \theta \\ \Delta|\mathbf{V}|\end{array}\right]$

where the Jacobian matrix $\left(\mathbf{J}_{\mathbf{I}}\right)$ has exactly the same structure of $\mathbf{J}$, for $n p q$ load buses and $n p v$ generator buses. Submatrices $\mathbf{J}_{1}$ $=-\partial \Delta \mathbf{G}(\boldsymbol{\theta},|\mathbf{V}|) / \partial \boldsymbol{\theta}, \mathbf{J}_{\mathbf{2}}=-\partial \Delta \mathbf{G}(\boldsymbol{\theta},|\mathbf{V}|) / \partial|\mathbf{V}|, \mathbf{J}_{\mathbf{3}}=-\partial \Delta \mathbf{H I}(\boldsymbol{\theta},|\mathbf{V}|) / \partial \boldsymbol{\theta}$ and $\mathbf{J}_{\mathbf{4}}-$ $=-\partial \Delta \mathbf{H I}(\boldsymbol{\theta},|\mathbf{V}|) / \partial|\mathbf{V}|$, which are obtained similarly to the SPF, are given by:

Submatrix $\mathbf{J}_{\mathbf{1}}$ :

$J_{1}(k, i)=-\left|V_{i}\right|\left|Y_{k i}\right| \sin \left(\phi_{k i}+\theta_{i}\right) \quad$ for $k \neq i$

$J_{1}(k, k)=-\left|V_{k}\right|\left|Y_{k k}\right| \sin \left(\phi_{k k}+\theta_{k}\right)+\frac{\left|S_{k}^{s p}\right|}{\left|V_{k}\right|} \sin \left(-\varphi_{k}+\theta_{k}\right)$

Submatrix $\mathbf{J}_{\mathbf{2}}$ :

$J_{2}(k, i)=\left|Y_{k i}\right| \cos \left(\phi_{k i}+\theta_{i}\right) \quad$ for $k \neq i$

$J_{2}(k, k)=\left|Y_{k k}\right| \cos \left(\phi_{k k}+\theta_{k}\right)+\frac{\left|S_{k}^{s p}\right|}{\left|V_{k}^{2}\right|} \cos \left(-\varphi_{k}+\theta_{k}\right)$

Submatrix $\mathbf{J}_{3}$ :

$J_{3}(k, i)=\left|V_{i}\right|\left|Y_{k i}\right| \cos \left(\phi_{k i}+\theta_{i}\right) \quad$ for $k \neq i$

$J_{3}(k, k)=\left|V_{k}\right|\left|Y_{k k}\right| \cos \left(\phi_{k k}+\theta_{k}\right)-\frac{\left|S_{k}^{s p}\right|}{\left|V_{k}\right|} \cos \left(-\varphi_{k}+\theta_{k}\right)$

Submatrix $\mathbf{J}_{\mathbf{4}}$ :

$J_{4}(k, i)=\left|Y_{k i}\right| \sin \left(\phi_{k i}+\theta_{i}\right) \quad$ for $k \neq i$

$J_{4}(k, k)=\left|Y_{k k}\right| \sin \left(\phi_{k k}+\theta_{k}\right)+\frac{\left|S_{k}^{s p}\right|}{\left|V_{k}^{2}\right|} \sin \left(-\varphi_{k}+\theta_{k}\right)$

In this formulation, as in the standard method, while the generated reactive power of a PV bus $k$ lies within the maximum and minimum limits, the respective balance equation is not included in (15) and the total number of equations is the same as in (1). On the other hand, differently from the standard method, the value of $Q_{g e n, k}$ affects the values of the correction vectors once it explicitly appear in $\Delta G_{k}$, as well as in the respective elements of $\mathbf{J}_{\mathbf{1}}$ and $\mathbf{J}_{2}$. This can be verified from the magnitude $\left|S_{k}^{s p}\right|$ and angle $\varphi_{k}$ of the net specified complex power injection specified at bus $k$ and present in the second element of the left hand side of (18) and (20). The appropriate representation of a PV bus in the current injection formulations has been addressed by several references, with the objective of improving the performance of the Newton current injection power flow. In [5] $Q_{g e n, k}$ is taken into account through an alternate adjustment. This adjustment is performed alternately with the solution iterative process [16]. During the iterative process $Q_{g e n, k}$ is kept unchanged, being recalculated before the next iteration by:

$Q_{\text {gen }, k}(\boldsymbol{\theta},|\mathbf{V}|)=Q_{\text {load }, k}^{s p}-\left|V_{k}\right| \sum_{i \in \kappa}\left|Y_{k i}\right|\left|V_{i}\right| \sin \left(\phi_{k i}-\theta_{k i}\right)$

Note that obtaining $Q_{g e n}$ requires no extra calculation effort, since this is already made when limit violations are checked. In [12] the unknown reactive power mismatch is treated as a dependent variable and an additional linearized equality constraint is introduced, imposing that $\Delta V_{k}=0$ from the first iteration. Due to convergence difficulties presented by the method because of the assumption of zero voltage mismatch, a new formulation has been proposed in [13]. The revised representation presented in [11] requires three equations for each PV bus. In [7], an equation of power injection mismatch is used to represent PV buses, improving the convergence characteristics and decreasing the number of equations to only one.

An approach, hereafter named as current injection power flow considering $Q_{\text {gen }, k}$ as variable (CIPFQ), considers the reactive power generated as a new variable $\left(Q_{G, k}\right)[6,12]$. As the voltage magnitude of a PV bus $k$ is known and does not appear in the unknown $\boldsymbol{\theta}$ and $\mathbf{V}$, its respective equation can be included in (15) and used to obtain its generated reactive power. In other words, it uses the simultaneous adjustment consisting on the inclusion of the equations and the new variables in the basic power flow problem [16]. In this augmented formulation, for each generation bus $k$ (PV) the imaginary component of the complex current mismatch $\left(\Delta H I_{k}(\boldsymbol{\theta},|\mathbf{V}|)\right)$ is considered in (15) and the linearization of (15) yields:

$$
\left[\begin{array}{c}
\Delta \mathbf{G} \\
\Delta \mathbf{H I}
\end{array}\right]=-\mathbf{J}_{\mathbf{I}}^{\prime}\left[\begin{array}{c}
\Delta \theta \\
\Delta|\mathbf{V}| \\
\Delta \mathbf{Q}_{\mathbf{G}}
\end{array}\right]=\left[\begin{array}{lll}
\mathbf{J}_{1} & \mathbf{J}_{2} & \mathbf{J}_{2}^{\prime} \\
\mathbf{J}_{3} & \mathbf{J}_{\mathbf{4}} & \mathbf{J}_{4}^{\prime}
\end{array}\right]\left[\begin{array}{c}
\Delta \theta \\
\Delta|\mathbf{V}| \\
3 \Delta \mathbf{Q}_{\mathbf{G}}
\end{array}\right]
$$

Note that in $\mathbf{J}_{\mathbf{2}}$ and $\mathbf{J}_{\mathbf{4}}$, the elements corresponding to the respective derivatives with respect to voltages magnitudes of PV buses do not appear, since they are specified. In $\mathbf{J}_{\mathbf{2}}$ and $\mathbf{J}_{\mathbf{4}}$ all elements are zero except those corresponding to PV bus $k$. In case bus $k$ is PV, the respective values are given by:

$$
\begin{aligned}
& J_{2}^{\prime}(k, k)=-\sin \left(\theta_{k}\right) /\left|V_{k}\right| \\
& J_{4}^{\prime}(k, k)=\cos \left(\theta_{k}\right) /\left|V_{k}\right|
\end{aligned}
$$

Differently from Jacobian matrices $\mathbf{J}$ and $\mathbf{J}_{\mathbf{I}}$, for $n p q$ load buses and $n p v$ generator buses the order of the matrix $\mathbf{J}_{\mathbf{I}}^{\prime}$ is (2npq $+2 n p v)$ by $(2 n p q+2 n p v)$. In CIPFQ as well as in $[6,12,13]$, the matrix dimension increased with the number of PV buses involved. Note that this formulation is somewhat different from that presented in $[4,12,13]$, which uses current injection mismatches with reactive mismatch $\Delta Q$ as a dependent variable, together with a voltage magnitude constraint equation both written in rectangular coordinates. It is also different from the hybrid formulation of current injection method written in polar coordinates presented in [6], which uses a dependent variable $\Delta Q$ for each PV bus associated to an active power mismatch equation.

In the new alternative formulation proposed in this paper, named as modified current injection power flow (MCIPF), $Q_{\text {gen }, k}$ is a function of the state variables $\boldsymbol{\theta}$ and $|\mathbf{V}|$. Hence, the elements of submatrices $\mathbf{J}_{\mathbf{1}}$ and $\mathbf{J}_{\mathbf{2}}$, given by (17)(20), are now obtained as follows: 
Submatrix $\mathbf{J}_{\mathbf{1}}$ :

$$
\begin{aligned}
J_{1}(k, i)= & -\left|V_{i}\right|\left|Y_{k i}\right| \sin \left(\phi_{k i}+\theta_{i}\right)+\left|V_{i}\right|\left|Y_{k i}\right| \cos \left(\phi_{k i}+\theta_{i}-\theta_{k}\right) \\
& \times \sin \left(\theta_{k}\right)
\end{aligned}
$$

$$
\begin{aligned}
J_{1}(k, k)= & -\left|V_{k}\right|\left|Y_{k k}\right| \sin \left(\phi_{k k}+\theta_{k}\right)+\frac{\left|S_{k}^{s p}\right|}{\left|V_{k}\right|} \sin \left(-\varphi_{k}+\theta_{k}\right) \\
& -\left[\frac{\left(P_{g e n, k}^{s p}-P_{\text {load }, k}^{s p}\right)}{\left|V_{k}\right|}-\left|V_{k}\right|\left|Y_{k k}\right| \cos \left(\phi_{k k}\right)\right] \sin \left(\theta_{k}\right)
\end{aligned}
$$

Submatrix $\mathbf{J}_{\mathbf{2}}$ :

$$
\begin{aligned}
J_{2}(k, i)= & \left|Y_{k i}\right| \cos \left(\phi_{k i}+\theta_{i}\right)+\left|Y_{k i}\right| \sin \left(\phi_{k i}+\theta_{i}-\theta_{k}\right) \sin \left(\theta_{k}\right) \\
J_{2}(k, k)= & \left|Y_{k k}\right| \cos \left(\phi_{k k}+\theta_{k}\right)+\left|\frac{S_{k}^{s p}}{V_{k}^{2}}\right| \cos \left(-\varphi_{k}+\theta_{k}\right) \\
& -\left[\frac{\left(Q_{\text {gen }, k}-Q_{\text {load }, k}^{s p}\right)}{\left|V_{k}^{2}\right|}-\left|Y_{k k}\right| \sin \left(\phi_{k k}\right] \sin \left(\theta_{k}\right)\right.
\end{aligned}
$$

Note that in $\mathbf{J}_{2}$, the diagonal elements $J_{2}(k, k)$ corresponding to the respective derivatives with respect to voltages magnitudes of PV buses do not appear, since their voltage magnitudes are specified. Likewise, even though the elements of $J_{3}$ and $J_{4}$ are also influenced by the unknown generated reactive power, their respective equations $\left(\Delta H I_{k}(\boldsymbol{\theta},|\mathbf{V}|)\right.$ are not enforced in the polar coordinates. So, the elements of submatrices $\mathbf{J}_{\mathbf{3}}$ and $\mathbf{J}_{\mathbf{4}}$ do not change, since they correspond to derivatives of equations related to PQ buses. The advantage of this formulation is that the structure and size of the Jacobian matrix remain the same as in (16), except the elements corresponding to the PV buses, which have their values changed in submatrices $\mathbf{J}_{\mathbf{1}}$ and $\mathbf{J}_{\mathbf{2}}$. This is shown by the new elements of the left hand sides of (29)(32), which correspond to the partial derivatives of $\Delta G_{k}(\boldsymbol{\theta},|\mathbf{V}|)$ with respect to $\boldsymbol{\theta}$ and $\mathbf{V}$ taking into account that $Q_{g e n, k}(\boldsymbol{\theta}, \mathbf{V})$ as defined in (25) is present in the magnitude $\left|S_{k}^{s p}\right|$ and angle $\varphi_{k}$ of the net specified complex power injection specified at bus $k$. In the case of the standard method, the new representation of PV buses adds one term for (29) and (31), and two terms in (30). On the other hand, as will be confirmed by the simulations, the convergence of the iterative process is also improved, since the elements of sub-matrices $\mathbf{J}_{\mathbf{1}}$ and $\mathbf{J}_{\mathbf{2}}$ are more accurately calculated, as they now take into account the influence of generated reactive power variation when obtaining the correction vectors $(\Delta \boldsymbol{\theta}$ and $\Delta|\mathbf{V}|)$. Furthermore, as discussed in [15], the performance of Newton's method is closely associated with the degree of nonlinearity of the power flow equations. The more linear it is, the better the performance. Comparing Eqs. (1) and (14), one can see that, unlike (1), Eq. (14) has only one term $\left(\left|S_{k}^{s p}\right| /\left|V_{k}\right|\right)$ on the right-hand sides that is not linear in $\left|V_{k}\right|$. Besides, each off-diagonal element of the power flow formulation based on current injection requires one less multiplication than the standard power flow (SPF), and then a smaller numbers of FLOPs (floatingpoint operations) [5], as will be confirmed in the next section.

\section{Test results}

The effectiveness of the proposed methodology is demonstrated through numerical tests that were conducted for the 57-, 118- and 300-bus IEEE test systems and for two realistic Brazilian systems of 638 and 787 buses, corresponding to parts of the South-Southeast Brazilian system. For all methods the maximum absolute value of the power mismatch vector $\mathbf{R}=\left[\Delta \mathbf{P}^{\mathrm{T}} \Delta \mathbf{Q}^{\mathrm{T}}\right]^{\mathrm{T}}\left(\|\mathbf{R}\|_{\infty}=\max \left\{\left|R_{i}\right|\right\}\right)$ is adopted as the convergence criterion. The convergence tolerance adopted was $10^{-5}$ p.u. Reactive power generation limits at PV buses are enforced in all methods.
For all tests, the iterative power flow process starts from the respective power system data files and the total mismatch is used to illustrate the iterative process convergence. This mismatch is defined as the sum of the absolute values of the real and reactive power mismatches. In order to highlight the convergence difficulties of the Newton-Raphson method, a maximum of 40 iterations was adopted in the simulations. The performance assessment is carried out considering different $\mathrm{R} / \mathrm{X}$ transmission line ratios and loading conditions. For the $\mathrm{R} / \mathrm{X}$ ratio tests, parameters $\mathrm{R}$ and $\mathrm{X}$ of all branches varied according to the multipliers shown in the first columns of the respective tables. For the loading tests, the real and reactive loads and real power generations varied according to the multipliers (loading factor, $\lambda$ ) shown in the first columns of the respective tables.

\subsection{Performance of the methods for the IEEE test systems}

Tables 1 and 2 show the numbers of iterations of the methods for the 57- and 118-bus IEEE test systems, considering different $\mathrm{R} / \mathrm{X}$ ratios. For both systems, all methods present a low number of iterations, except for CIFP, that shows a significant increase in the number of iterations required for the 118-bus system. The weak performance in this case is due to the presence of a larger number of PV buses. Note for both systems that the methods do not converge for $\mathrm{R}$ multipliers equal to or greater than four.

Fig. 1(a) depicts the trajectories of total power mismatches for the $3 \times \mathrm{R} / 1 \times \mathrm{X}$ condition of the 118 -bus system. CIPF needs 18 iterations to obtain the solution, whereas the other methods require only five iterations for convergence. From the analysis of total mismatch evolution one can see that a slight oscillatory behavior occurs at the second iteration, whose value decreases slowly in the next iterations.

Fig. 1(b) and (c) shows the converged states $(\boldsymbol{\theta},|\mathbf{V}|)$ obtained by the methods, for the IEEE 118-bus system. At the end of the iterative process, all methods converge to the same solution.

Tables 3 and 4 present the numbers of iterations needed by the methods to obtain the solution starting from the state from the data file and considering different loading conditions and a fixed $\mathrm{R} / \mathrm{X}$ ratio of $1 \times \mathrm{R} / 1 \times \mathrm{X}$. The loading values presented in the first column of Tables 3 and 4 were set from the knowledge of the maximum loading value $\left(\lambda_{\max }\right)$ of each system, 1.5972 and 1.8664 p.u. for the 57-bus and 118-bus systems, respectively. The maximum loading values and the P-V curves were previously obtained using the continuation power flow presented in [17]. Loads are modeled

Table 1

IEEE 57-bus system: Performance for different R/X ratios.

\begin{tabular}{lllll}
\hline $\mathrm{R} / \mathrm{X}$ ratios & $\mathrm{SPF}$ & $\mathrm{CIPF}$ & $\mathrm{CIPFQ}$ & $\mathrm{MCIPF}$ \\
\hline $1.0 \times \mathrm{R} / 0.5 \times \mathrm{X}$ & 4 & 4 & 3 & 3 \\
$1.0 \times \mathrm{R} / 1.0 \times \mathrm{X}$ & 4 & 5 & 4 & 4 \\
$2.0 \times \mathrm{R} / 1.0 \times \mathrm{X}$ & 5 & 6 & 4 & 4 \\
$3.0 \times \mathrm{R} / 1.0 \times \mathrm{X}$ & 8 & 8 & 5 & 5 \\
$4.0 \times \mathrm{R} / 1.0 \times \mathrm{X}$ & $\mathrm{NC}$ & $\mathrm{NC}$ & $\mathrm{NC}$ & $\mathrm{NC}$ \\
\hline
\end{tabular}

NC- no convergence or divergence for a maximum iteration number of 40 .

Table 2

IEEE 118-bus system: Performance for different R/X ratios.

\begin{tabular}{lllll}
\hline $\mathrm{R} / \mathrm{X}$ ratios & $\mathrm{SPF}$ & $\mathrm{CIPF}$ & $\mathrm{CIPFQ}$ & $\mathrm{MCIPF}$ \\
\hline $1.0 \times \mathrm{R} / 0.5 \times \mathrm{X}$ & 5 & 8 & 5 & 5 \\
$1.0 \times \mathrm{R} / 1.0 \times \mathrm{X}$ & 5 & 7 & 5 & 5 \\
$2.0 \times \mathrm{R} / 1.0 \times \mathrm{X}$ & 5 & 10 & 5 & 5 \\
$3.0 \times \mathrm{R} / 1.0 \times \mathrm{X}$ & 5 & 18 & 5 & 5 \\
$4.0 \times \mathrm{R} / 1.0 \times \mathrm{X}$ & $\mathrm{NC}$ & $\mathrm{NC}$ & $\mathrm{NC}$ & $\mathrm{NC}$ \\
\hline
\end{tabular}




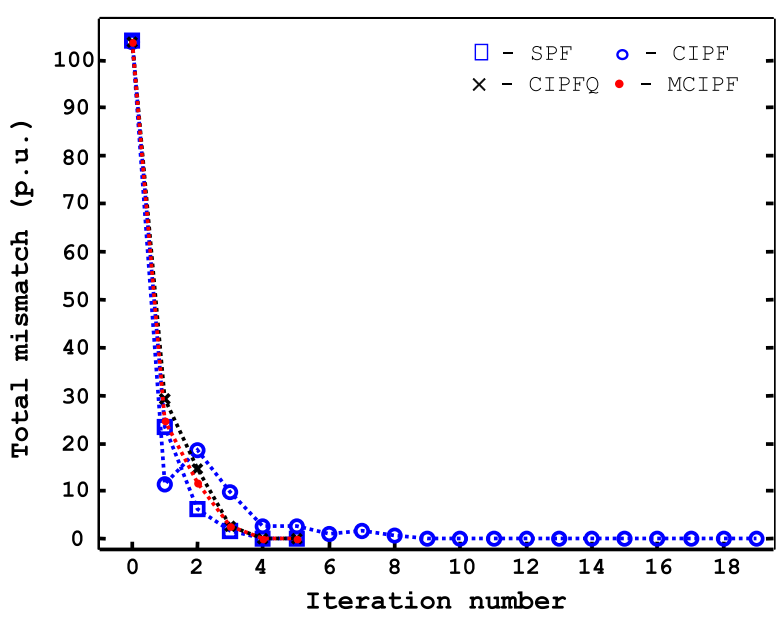

(a)

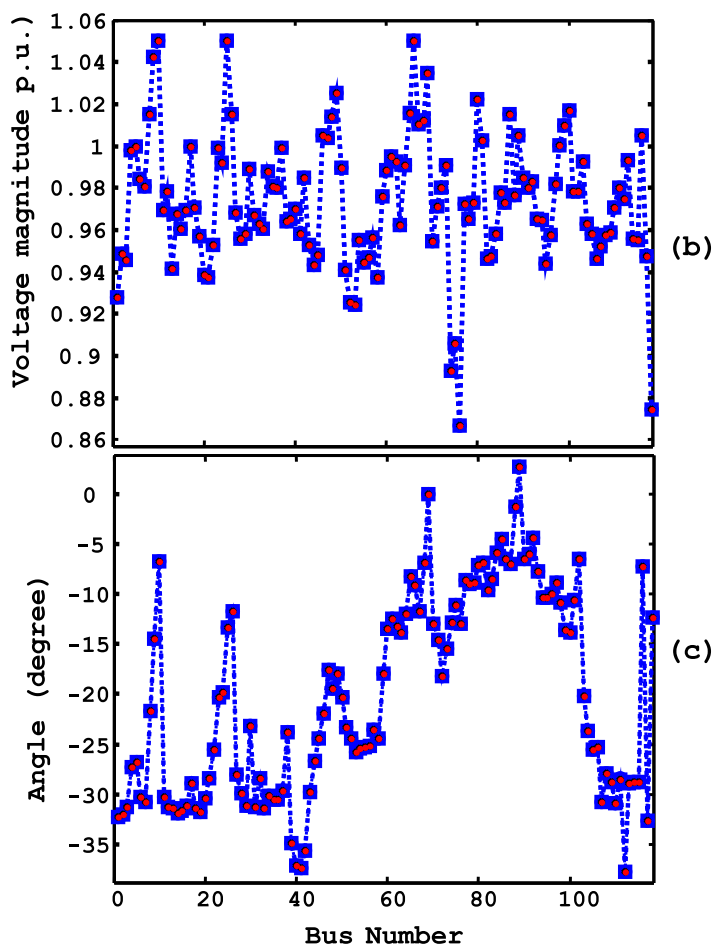

Fig. 1. IEEE 118-bus system: (a) evolution of total mismatches of each one of the methods, (b) voltage and (c) angle profiles, for the $3 \times \mathrm{R} / 1 \times \mathrm{X}$ ratio.

Table 3

IEEE 57-bus system: Performance for different loading conditions.

\begin{tabular}{lllll}
\hline$\lambda$ (p.u.) & SPF & CIPF & CIPFQ & MCIPF \\
\hline 1.0 & 4 & 5 & 4 & 4 \\
1.4 & 5 & 5 & 4 & 4 \\
1.5 & 5 & 6 & 4 & 4 \\
1.58 & 6 & 5 & 5 & 5 \\
1.596 & 8 & 7 & 7 & 7 \\
1.6 & NC & NC & NC & NC \\
\hline
\end{tabular}

Table 4

IEEE 118-bus system: Performance for different loading conditions.

\begin{tabular}{lllll}
\hline$\lambda$ (p.u.) & SPF & CIPF & CIPFQ & MCIPF \\
\hline 1.0 & 5 & 7 & 5 & 5 \\
1.4 & 5 & 11 & 5 & 5 \\
1.7 & 6 & 17 & 6 & 6 \\
1.86 & 8 & NC & 8 & 9 \\
1.865 & 9 & NC & 9 & 10 \\
1.9 & NC & NC & NC & NC \\
\hline
\end{tabular}

as constant power and loading factor $\lambda$ is applied to the real and reactive loads, considering a constant power factor [1,2]. The loading factor is also used to provide an equivalent increase in active power generation. The voltage magnitudes of the critical bus obtained by each method are plotted on the respective P-V curves, to confirm that the converged state obtained by all methods is the same, as shown in Fig. 2(a) and (b).

Table 2 shows that all methods presented similar performances, including an expected increase in the number of iterations as the system approaches the maximum loading point. In spite of the singularities of Jacobian matrices at the maximum loading point $\left(\lambda_{\max }\right)$, operating points very close to it can be obtained starting from the system data file condition showing the robustness of the methods. Table 3 shows that similar performances are presented by the methods for the 118-bus system, except CIPF [5], that fails for values of $\lambda$ larger or equal to 1.7 p.u. As expected, all of them fail to loadings slightly higher than $\lambda_{\max }$.

Table 5 shows the performance of the IEEE 300-bus system for different $\mathrm{R} / \mathrm{X}$ factors. All methods present similar performance, failing to converge for multiplier $\mathrm{R}$ equal to or greater than 1.473. Note that CIPF requires more iterations than the other methods. The trajectories of total power mismatches for $\mathrm{R} / \mathrm{X}$ condition of $1.472 \times \mathrm{R} / 1 \times \mathrm{X}$, for which there is convergence, is shown in Fig. 3(a). Fig. 3(b) shows the P-V curve, from which it can be clearly seen that the new corresponding maximum loading value is equal to 1.0 p.u. and this is the main reason why the methods do not converge.

The performances for different loading conditions are presented in Table 6. The maximum loading point for this system $\left(\lambda_{\max }=1.0553\right.$ p.u. $)$ was obtained by a continuation power flow. Thus, the methods do not converge to slightly higher values than $\lambda_{\text {max }}$

\subsection{Performance of the methods for the realistic Brazilian systems}

Tables 7-10 show simulation results for two realistic large systems corresponding to parts of the south-southeast Brazilian system, respectively 638-bus, 1276-branch and 787-bus, 1395branch systems. Tables 7 and 8 show the effect of $\mathrm{R} / \mathrm{X}$ ratio changes, while Tables 9 and 10 of the loading factor increments. These systems are heavily loaded, particularly the 638-bus, which represents a very stressed operating condition, i.e., its maximum loading point is very close to the base case. The respective exact maximum loadings of these systems are a little bit larger than 1.0087 and 1.1273 p.u. While the methods require a reasonable number of iterations to converge, CIPF presents a larger number of iterations, not converging for most cases analyzed.

Fig. 4(a) and (b) shows the evolution of the total mismatches for the base case $(\lambda=1.0$ p.u. and $1.0 \times \mathrm{R} / 1.0 \times \mathrm{X})$ for the 638 - and 787 -bus systems, respectively. The methods show the same convergence characteristics, except CIPF. There is a great reduction in the total mismatch magnitudes in the first iterations, regardless of the system' size, a fact already expected for the NewtonRaphson's method.

Fig. 4(c) shows the P-V curve for the critical bus of the 638-bus system for $1.098 \times \mathrm{R} / 1.0 \times \mathrm{X}$, from which the respective maximum loading is equal to 1.0 p.u. Therefore, this is the maximum multiplier that results in a feasible solution for the load flow problem.

Table 11 shows the CPU times for the methods, except CIPF, for the most critical condition of ratio R/X and loading condition. For cases where the numbers of iterations for convergence differ, the calculation of the normalized CPU time considered the smallest number of iterations for all three methods. Their values were normalized by the respective CPU times of the SPF method (third column). Compared to SPF, MCIPF results in an average CPU time reduction of $2 \%$, while CIPFQ results in an increase of $2 \%$. 


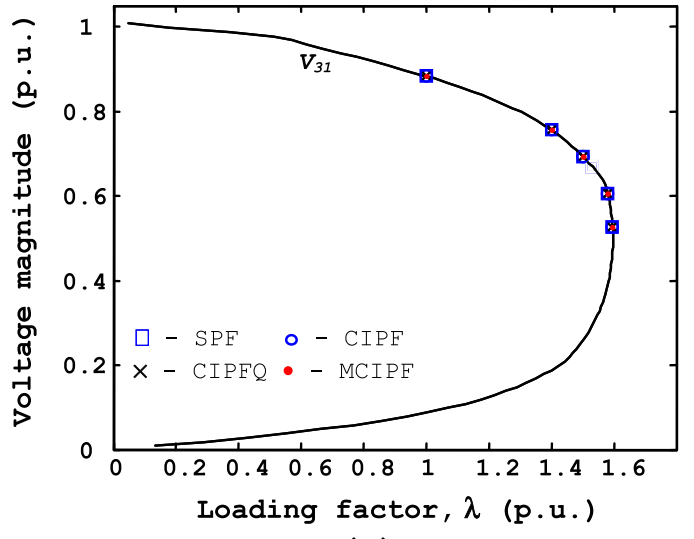

(a)

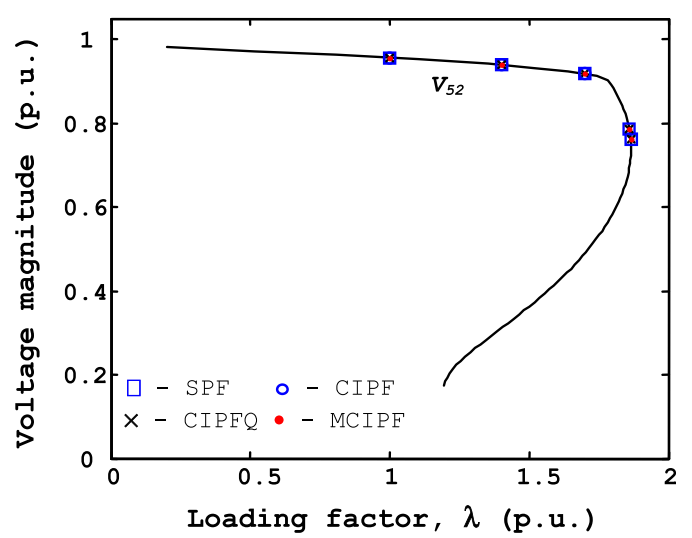

(b)

Fig. 2. P-V curve for the critical bus of (a) 57 -bus and (b) 118 -bus system, for the base case $(1 \times R / 1 \times X)$.

Table 5

IEEE 300-bus system: Performance or different R/X ratios.

\begin{tabular}{lllll}
\hline $\mathrm{R} / \mathrm{X}$ ratio & $\mathrm{SPF}$ & $\mathrm{CIPF}$ & $\mathrm{CIPFQ}$ & $\mathrm{MCIPF}$ \\
\hline $1.0 \times \mathrm{R} / 0.5 \times \mathrm{X}$ & 5 & 7 & 6 & 6 \\
$1.0 \times \mathrm{R} / 1.0 \times \mathrm{X}$ & 4 & 10 & 4 & 4 \\
$1.2 \times \mathrm{R} / 1.0 \times \mathrm{X}$ & 5 & 10 & 5 & 5 \\
$1.4 \times \mathrm{R} / 1.0 \times \mathrm{X}$ & 6 & 11 & 5 & 5 \\
$1.47 \times \mathrm{R} / 1.0 \times \mathrm{X}$ & 7 & 13 & 7 & 7 \\
$1.472 \times \mathrm{R} / 1.0 \times \mathrm{X}$ & 8 & 13 & 8 & 8 \\
$1.473 \times \mathrm{R} / 1.0 \times \mathrm{X}$ & $\mathrm{NC}$ & $\mathrm{NC}$ & $\mathrm{NC}$ & $\mathrm{NC}$
\end{tabular}
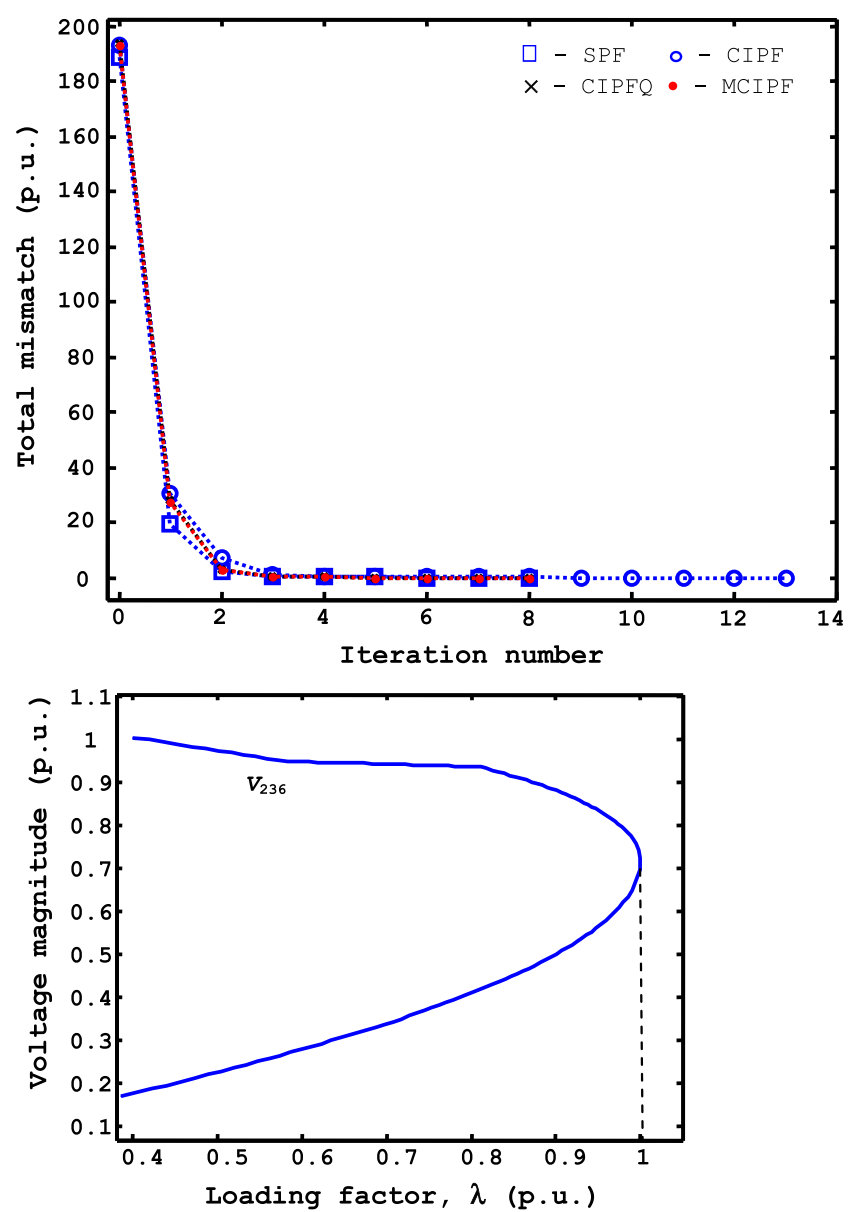

Fig. 3. IEEE 300 -bus system for $1.472 \times \mathrm{R} / 1 \times \mathrm{X}$ : (a) evolution of total mismatches and (b) P-V curve of critical bus.
Table 6

IEEE 300-bus system: Performance for different loading conditions.

\begin{tabular}{lllll}
\hline$\lambda$ (p.u.) & SPF & CIPF & CIPFQ & MCIPF \\
\hline 1.0 & 4 & 10 & 4 & 4 \\
1.02 & 5 & 10 & 5 & 5 \\
1.04 & 5 & 9 & 5 & 5 \\
1.05 & 6 & 10 & 6 & 6 \\
1.055 & 7 & 10 & 7 & 7 \\
1.056 & NC & NC & NC & NC \\
\hline
\end{tabular}

Table 7

IEEE 638-bus system: Performance for different R/X ratios.

\begin{tabular}{lllll}
\hline $\mathrm{R} / \mathrm{X}$ ratio & $\mathrm{SPF}$ & $\mathrm{CIPF}$ & $\mathrm{CIPFQ}$ & $\mathrm{MCIPF}$ \\
\hline $1.0 \times \mathrm{R} / 0.5 \times \mathrm{X}$ & 5 & 10 & 4 & 5 \\
$1.0 \times \mathrm{R} / 1.0 \times \mathrm{X}$ & 5 & $\mathrm{NC}$ & 4 & 4 \\
$1.08 \times \mathrm{R} / 1.0 \times \mathrm{X}$ & 6 & $\mathrm{NC}$ & 5 & 5 \\
$1.095 \times \mathrm{R} / 1.0 \times \mathrm{X}$ & 7 & $\mathrm{NC}$ & 6 & 6 \\
$1.098 \times \mathrm{R} / 1.0 \times \mathrm{X}$ & 9 & $\mathrm{NC}$ & 8 & 8 \\
$1.1 \times \mathrm{R} / 1.0 \times \mathrm{X}$ & $\mathrm{NC}$ & $\mathrm{NC}$ & $\mathrm{NC}$ & $\mathrm{NC}$ \\
\hline
\end{tabular}

Table 8

IEEE 787-bus system: Performance for different R/X ratios.

\begin{tabular}{lllll}
\hline $\mathrm{R} / \mathrm{X}$ ratio & $\mathrm{SPF}$ & $\mathrm{CIPF}$ & $\mathrm{CIPFQ}$ & $\mathrm{MCIPF}$ \\
\hline $1.0 \times \mathrm{R} / 0.5 \times \mathrm{X}$ & 4 & 9 & 4 & 4 \\
$1.0 \times \mathrm{R} / 1.0 \times \mathrm{X}$ & 3 & 14 & 3 & 3 \\
$2.0 \times \mathrm{R} / 1.0 \times \mathrm{X}$ & 4 & $\mathrm{NC}$ & 4 & 4 \\
$2.4 \times \mathrm{R} / 1.0 \times \mathrm{X}$ & 5 & $\mathrm{NC}$ & 5 & 5 \\
$2.48 \times \mathrm{R} / 1.0 \times \mathrm{X}$ & 7 & $\mathrm{NC}$ & 7 & 7 \\
$2.485 \times \mathrm{R} / 1.0 \times \mathrm{X}$ & 8 & $\mathrm{NC}$ & 8 & 8 \\
$2.486 \times \mathrm{R} / 1.0 \times \mathrm{X}$ & $\mathrm{NC}$ & $\mathrm{NC}$ & $\mathrm{NC}$ & $\mathrm{NC}$ \\
\hline
\end{tabular}

Table 9

IEEE 638-bus system: Performance for different loading conditions.

\begin{tabular}{lllll}
\hline$\lambda$ (p.u.) & SPF & CIPF & CIPFQ & MCIPF \\
\hline 1.000 & 5 & NC & 4 & 4 \\
1.002 & 5 & NC & 5 & 5 \\
1.005 & 5 & NC & 5 & 5 \\
1.008 & 6 & NC & 6 & 6 \\
1.0087 & 8 & NC & 7 & 7 \\
1.0088 & NC & NC & NC & NC \\
\hline
\end{tabular}


Table 10

IEEE 787-bus system: Performance for different loading conditions.

\begin{tabular}{lllll}
\hline$\lambda$ (p.u.) & SPF & CIPF & CIPFQ & MCIPF \\
\hline 1.0 & 3 & 14 & 3 & 3 \\
1.08 & 4 & 36 & 4 & 4 \\
1.10 & 5 & NC & 5 & 5 \\
1.1273 & 8 & NC & 8 & 8 \\
1.1274 & NC & NC & NC & NC \\
\hline
\end{tabular}
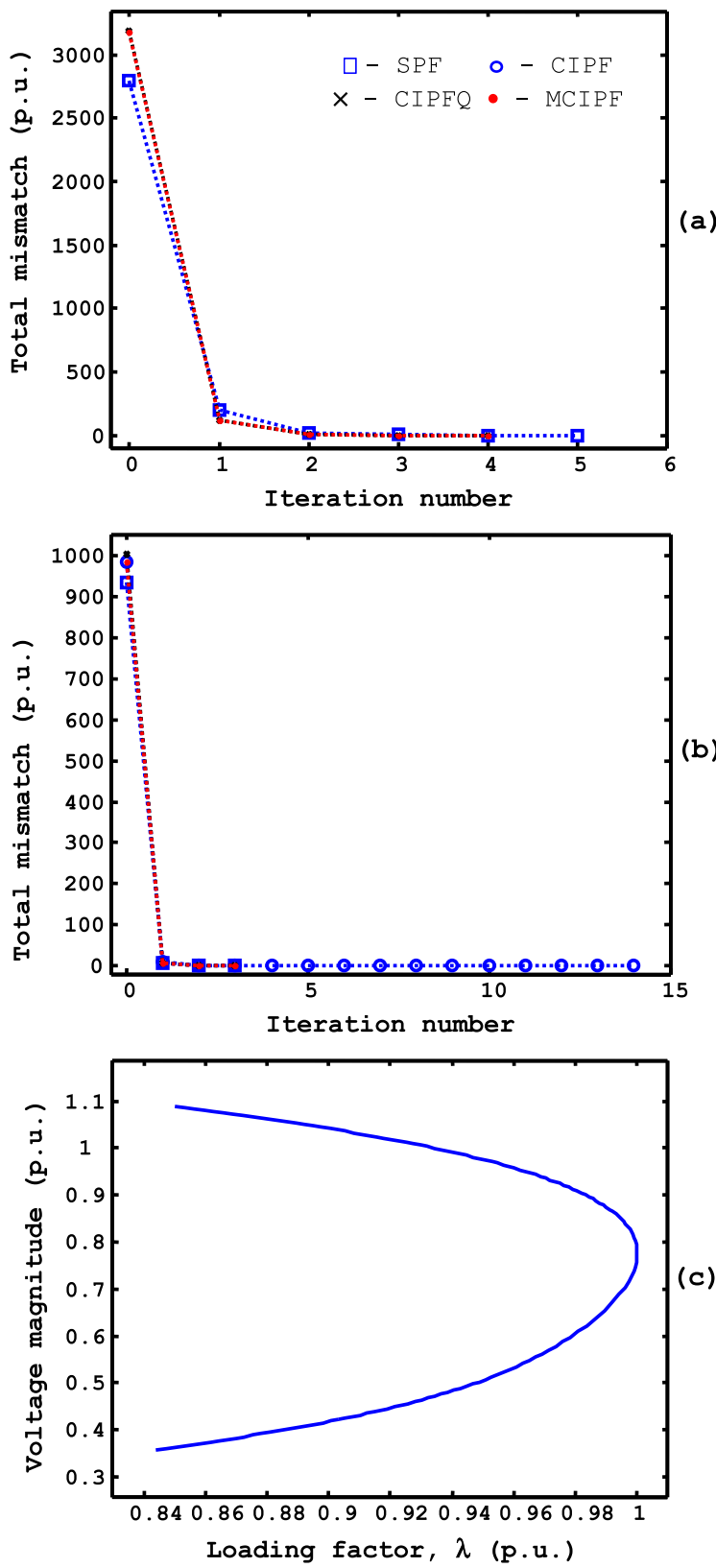

Fig. 4. Evolution of the total mismatches for the base case for (a) 638-bus system and (b) 787-bus system, and (c) P-V curve for the critical bus of the 638-bus system for $1.098 \times \mathrm{R} / 1.0 \times \mathrm{X}$.

Table 12 presents a comparison between the FLOPs per iteration required by MCIPF and SPF. It can be seen from table that the proposed method requires a slightly smaller number of FLOPs than SPF. It is important to note that this feature is still maintained, even when the long sums of diagonal submatrix elements of SPF are
Table 11

Performance of the methods - Normalized CPU times.

\begin{tabular}{llllllll}
\hline \multirow{2}{*}{ System } & \multirow{2}{*}{$\begin{array}{l}\text { Loading } \\
\text { Condition }\end{array}$} & \multicolumn{3}{c}{ Normalized CPU time (p.u.) } & & \multicolumn{2}{c}{ CPU Ratio (\%) } \\
& & SPF & MCIPF & CIPFQ & & MCIPF & CIPFQ \\
\hline 300 & 1.055 & 1.0000 & 0.9783 & 1.0249 & -2.17 & 2.49 \\
638 & 1.0087 & 1.0000 & 0.9726 & 1.0190 & -2.74 & 1.90 \\
787 & 1.1273 & 1.0000 & 0.9784 & 1.0221 & -2.16 & 2.21 \\
& $\mathrm{R} / \mathrm{X}$ ratio condition & & & & & \\
300 & $1.472 \times \mathrm{R} / 1.0 \times \mathrm{X}$ & 1.0000 & 0.9811 & 1.4920 & -1.90 & 1.49 \\
638 & $1.098 \times \mathrm{R} / 1.0 \times \mathrm{X}$ & 1.0000 & 0.9722 & 1.0074 & -2.78 & 0.74 \\
787 & $2.485 \times \mathrm{R} / 1.0 \times \mathrm{X}$ & 1.0000 & 0.9814 & 1.0312 & -1.87 & 3.12 \\
\hline
\end{tabular}

Table 12

Number of FLOPs per iteration.

\begin{tabular}{llll}
\hline $\mathrm{nPV}$ & System & SPF & MCIPF \\
\hline 68 & 300 & 225,019 & 208,949 \\
97 & 638 & 660,738 & 634,308 \\
111 & 787 & 751,586 & 708,475 \\
\hline
\end{tabular}

avoided and the new elements of the left hand sides of $(29)(32)$ take into account the dependence of $Q_{g e n, k}$ with the state variables $\theta$ and $|\mathrm{V}|$.

\section{Conclusion}

In this work the influence of three ways of treating generation (PV) buses are compared as far as the convergence characteristics of Newton-Raphson method for solving the power flow problem based in current injections considering polar coordinates. A comparison between power injection and current injection-based formulation in terms of polar coordinates is also shown.

The performance analyzes are carried out for the 57-, 118- and 300-bus IEEE test systems as well as two versions of the Brazilian interconnected system, with 638 and 787 buses. Different R/X ratios and loading conditions were taken into account. The results obtained with the proposed formulation showed that it is possible to improve the convergence characteristics of the current injection power flow model proposed in [5] in terms of number of iterations and CPU time. The convergence of the proposed process is improved since the elements of sub-matrices $\mathbf{J}_{\mathbf{1}}$ and $\mathbf{J}_{\mathbf{2}}$ are more accurately calculated, as they now take into account the influence of generated reactive power variation when obtaining the correction vectors $(\Delta \boldsymbol{\theta}$ and $\Delta|\mathbf{V}|)$. When compared to the conventional power injection method considering polar coordinates, this simple change in the calculation of the derivatives increases the efficiency of the proposed technique and proves that it is possible to obtain a reduction in computational time of current injection method written in polar coordinates, without loss of robustness.

\section{Acknowledgments}

The authors are grateful to the financial support provided by CNPq and CAPES (Brazilian Research Funding Agencies).

\section{Appendix A}

For a PV bus, the elements of the MCIPF submatrices $\mathbf{J}_{\mathbf{1}}$ and $\mathbf{J}_{\mathbf{2}}$ are obtained as follows. 


\section{A.1. Elements of submatrix $\boldsymbol{J}_{1}$}

The real component $\left(\Delta G_{k}(\boldsymbol{\theta},|\mathbf{V}|)\right)$ in (15) can be written as

$$
\begin{aligned}
\Delta G_{k}(\boldsymbol{\theta},|\mathbf{V}|)= & \frac{P_{k}^{s p}}{\left|V_{k}\right| s} \cos \left(\theta_{k}\right)+\frac{Q_{\text {gen }, k}(\boldsymbol{\theta},|\mathbf{V}|)-Q_{\text {load }, k}^{s p}}{\left|V_{k}\right|} \sin \left(\theta_{k}\right) \\
& -G_{k k}\left|V_{k}\right| \cos \left(\theta_{k}\right)+B_{k k}\left|V_{k}\right| \sin \left(\theta_{k}\right) \\
& -\sum_{i \in \Omega_{k}}\left|Y_{k i}\right|\left|V_{i}\right| \cos \left(\phi_{k i}+\theta_{i}\right)=0,
\end{aligned}
$$

where $\Omega_{k}$ is the set of buses directly connected to bus $k$ and $Q_{g e n, k}$ is given by (25). The derivatives of $\Delta G_{k}$ with respect to $\theta_{i}$ can be expressed as

$$
\begin{aligned}
J_{1}(k, i) & =-\partial \Delta G_{k} / \partial \theta_{i} \\
& =-\left|V_{i}\right|\left|Y_{k i}\right| \sin \left(\phi_{k i}+\theta_{i}\right)-\frac{\sin \left(\theta_{k}\right)}{\left|V_{k}\right|} \partial Q_{g e n, k}(\boldsymbol{\theta},|\mathbf{V}|) / \partial \theta_{i} \\
& =-\left|V_{i}\right|\left|Y_{k i}\right| \sin \left(\phi_{k i}+\theta_{i}\right)+\left|V_{i}\right|\left|Y_{k i}\right| \cos \left(\phi_{k i}+\theta_{i}-\theta_{k}\right) \sin \left(\theta_{k}\right),
\end{aligned}
$$

while the derivatives of $\Delta G_{k}$ with respect to $\theta_{k}$ can be expressed as $J_{1}(k, k)=-\partial \Delta G_{k} / \partial \theta_{k}$

$$
\begin{aligned}
= & -\frac{\left(-P_{k}^{s p} \sin \left(\theta_{k}\right)+Q_{k}^{s p} \cos \left(\theta_{k}\right)\right)}{\left|V_{k}\right|}-\left|V_{k}\right|\left(G_{k k} \sin \left(\theta_{k}\right)+B_{k k}\right. \\
& \left.\times \sin \left(\theta_{k}\right)\right)-\frac{\sin \left(\theta_{k}\right)}{\left|V_{k}\right|} \partial Q_{g e n, k}(\boldsymbol{\theta},|\mathbf{V}|) / \partial \theta_{k} .
\end{aligned}
$$

The derivatives of $Q_{\text {gen, } k}$ with respect to $\theta_{k}$ is given by

$$
\begin{aligned}
\partial Q_{\text {gen }, k}(\boldsymbol{\theta},|\mathbf{V}|) / \partial \theta_{k} & =\left|V_{k}\right| \sum_{i \in \Omega_{k}}\left|Y_{k i}\right|\left|V_{i}\right| \cos \left(\phi_{k i}-\theta_{k}+\theta_{i}\right) \\
& =P_{k}(\boldsymbol{\theta},|\mathbf{V}|)-\left|V_{k}\right|^{2}\left|Y_{k k}\right| \cos \left(\phi_{k k}\right) .
\end{aligned}
$$

By plugging the equation above into the expression of $J_{1}(k, k)$ one gets

$$
\begin{aligned}
J_{1}(k, k)= & -\left|V_{k}\right|\left|Y_{k k}\right| \sin \left(\phi_{k k}+\theta_{k}\right)+\frac{\left|S_{k}^{s p}\right|}{\left|V_{k}\right|} \sin \left(-\varphi_{k}+\theta_{k}\right) \\
& -\left[\frac{\left(P_{g e r, k}^{s p}-P_{\text {carga }, k}^{s p}\right)}{\left|V_{k}\right|}-\left|V_{k}\right|\left|Y_{k k}\right| \cos \left(\phi_{k k}\right)\right] \sin \left(\theta_{k}\right),
\end{aligned}
$$

where $P_{k}(\boldsymbol{\theta},|\mathbf{V}|)$ is assumed equal to $P_{k}^{s p}$.

\section{A.2. Elements of submatrix $\boldsymbol{J}_{2}$}

The derivatives of $\Delta G_{k}$ with respect to $V_{i}$ can be expressed as

$$
\begin{aligned}
J_{2}(k, i) & =-\partial \Delta G_{k} / \partial V_{i} \\
& =\left|Y_{k i}\right| \cos \left(\phi_{k i}+\theta_{i}\right)-\frac{\sin \left(\theta_{k}\right)}{\left|V_{k}\right|} \partial Q_{g e n, k}(\boldsymbol{\theta},|\mathbf{V}|) / \partial V_{i} \\
& =\left|Y_{k i}\right| \cos \left(\phi_{k i}+\theta_{i}\right)+\left|Y_{k i}\right| \sin \left(\phi_{k i}+\theta_{i}-\theta_{k}\right) \sin \left(\theta_{k}\right) .
\end{aligned}
$$

while the derivatives of $\Delta G_{k}$ with respect to $V_{k}$ can be expressed as

$$
\begin{aligned}
J_{2}(k, k)= & -\partial \Delta G_{k} / \partial V_{k} \\
= & \frac{\left(P_{k}^{s p} \cos \left(\theta_{k}\right)+Q_{k}^{s p} \sin \left(\theta_{k}\right)\right)}{\left|V_{k}\right|^{2}}+\left(G_{k k} \cos \left(\theta_{k}\right)-B_{k k} \sin \left(\theta_{k}\right)\right) \\
& -\frac{\sin \left(\theta_{k}\right)}{\left|V_{k}\right|} \partial Q_{\text {gen }, k}(\boldsymbol{\theta},|\mathbf{V}|) / \partial V_{k} .
\end{aligned}
$$

The derivatives of $Q_{g e n, k}$ with respect to $V_{k}$ is given by

$$
\begin{aligned}
\partial Q_{g e n, k}(\boldsymbol{\theta},|\mathbf{V}|) / \partial\left|V_{k}\right|= & -2\left|V_{k}\right|\left|Y_{k k}\right| \sin \left(\phi_{k k}\right)-\sum_{i \in \Omega_{k}}\left|Y_{k i}\right|\left|V_{i}\right| \sin \left(\phi_{k i}-\theta_{k}\right. \\
& \left.+\theta_{i}\right) \\
= & Q_{k}(\boldsymbol{\theta},|\mathbf{V}|) /\left|V_{k}\right|-\left|V_{k}\right|\left|Y_{k k}\right| \sin \left(\phi_{k k}\right) .
\end{aligned}
$$

By plugging the equation above into the expression of $J_{2}(k, k)$ one gets

$$
\begin{aligned}
J_{2}(k, k)= & -\partial \Delta G_{k} / \partial V_{k} \\
= & \left|Y_{k k}\right| \cos \left(\phi_{k k}+\theta_{k}\right)+\left|\frac{S_{k}^{s p}}{V_{k}^{2}}\right| \cos \left(-\varphi_{k}+\theta_{k}\right) \\
& -\left[\frac{\left(Q_{g e r, k}-Q_{\text {carga }, k}^{s p}\right)}{\left|V_{k}^{2}\right|}-\left|Y_{k k}\right| \sin \left(\phi_{k k}\right)\right] \sin \left(\theta_{k}\right) .
\end{aligned}
$$

where $Q_{k}(\boldsymbol{\theta},|\mathbf{V}|)$ is assumed equal to $Q_{k}^{s p}$.

\section{References}

[1] Ajjarapu V. Computational techniques for voltage stability assessment and control. Power electronics and power systems series. New York: Springer; 2010.

[2] Kundur P. Power system stability and control. New York: McGraw-Hill; 1993. 1176 p..

[3] El-Hawary ME. Electrical power systems: design and analysis. Revised printing, vol. 2. John Wiley \& Sons; 1995. 799 p..

[4] Da Costa VM, Martins N, Pereira JLR. An augmented Newton-Raphson power flow formulation based on current injections. Electr Power Energy Syst 2001;23:305-12.

[5] Kulworawanichpong T. Simplified Newton-Raphson power-flow solution method. Electr Power Energy Syst 2010;32:551-8.

[6] Zhang Y, Lu Y. A novel newton current equation method on power flow analysis in microgrid. In: Power \& energy society general meeting; 2009.

[7] Kamel S, Abdel-Akher M, Jurado F. Improved NR current injection load flow using power mismatch representation of PV bus. Electr Power Energy Syst 2013;53(1):64-8.

[8] Kamel S, Jurado F, Chen Z, Abdel-Akher M, Ebeed M. Developed generalised unified power flow controller model in the Newton-Raphson power-flow analysis using combined mismatches method. IET Gener, Transm Distrib 2016;10(9):2177-84.

[9] Gómez-Expósito A, Romero-Ramos E, Dzafic I. Hybrid real-complex current injection-based load flow formulation. Electr Power Syst Res 2015;119:237-46.

[10] Expósito AG, Ramos ER. Augmented rectangular load flow model. IEEE Trans Power Syst 2002;17(2):271-6.

[11] Kamel S, Abdel-Akher M, El-Nemr MK. A new technique to improve voltage controlled nodes (PV nodes) in the current injection Newton-Raphson powerflow analysis. In: 45th International Universities Power Engineering Conference (UPEC); 2010.

[12] Da Costa VM, Martins N, Pereira JLR. Developments in the Newton-Raphson power flow formulation based on current injections. IEEE Trans Power Syst 1999;14(4):1320-6.

[13] Garcia PAN, Pereira JLR, Carneiro Jr S, Vinagre MP, Gomes FV. Improvements in the representation of PV buses on three-phase distribution power flow. IEEE Trans Power Deliv 2004;19(2):894-6.

[14] Dommel HW, Tinney WF, Powell WL. Further developments in Newton's method for power system applications. In: IEEE winter power meeting, conference paper no. 70 CP 161-PWR. New York; 1970.

[15] Stott B. Review of load-flow calculation methods. Proc IEEE 1974;62:916-29.

[16] Monticelli AJ. Power flow in electric networks. São Paulo (Brazil): Edgard Blucher; 1983 [in Portuguese].

[17] Bonini Neto A, Alves DA. An improved parameterization technique for the continuation power flow. In: Transmission and distribution conference and exposition, IEEE PES. New Orleans (LA, USA); 2010. p. 1-6. 